\title{
Evidence not strong enough to advocate powered toothbrushes over manual for orthodontic patients
}

\author{
Are powered toothbrushes more effective than manual brushes in reducing \\ gingival inflammation when patients are undergoing fixed appliance \\ orthodontic treatment?
}

\section{Kaklamanos EG, Kalfas S.}

Meta-analysis on the effectiveness of powered toothbrushes for orthodontic patients. Am J Orthod Dentofacial Orthop 2008; 133:18\&,e1-14

Data sources Studies were sourced using Medline, Embase, Cumulative Index to Nursing and Allied Health Literature, Cochrane Library, Institute for Scientific Information proceedings, Cambridge Scientific Abstracts, UMI Proquest, Trials Central (www.trialscentral.org) and the metaregister of controlled trials (www.controlled-trials.coms), along with the references of identified articles. No restrictions were placed on year of publication, publication status or language of the retrieved trials.

Study selection Randomised controlled trials were selected that compared powered and manual toothbrushes and involved participants of any age who wore fixed appliances, and which reported outcome measures quantifying gingival inflammation. Cross-over studies with a washout period of at least 1 month between experimental periods were also included. Split-mouth studies and trials involving interventions that combined toothbrushing with the use of antimicrobial mouthrinses, irrigation devices, or interdental cleansing and those of less than 4 weeks' duration were excluded.

Data extraction and synthesis Initial assessment and data abstraction was carried out by two reviewers independently. The quality of the trials was evaluated by assessing randomisation, allocation concealment, blinding of the examiner, description of losses, and the use of intention to treat analyses. Trials were divided into categories depending on the mode of action of the powered toothbrush. The weighted mean difference with $95 \%$ confidence intervals was used to express the comparative treatment effect. The random-effects method for meta-analysis was used to combine treatment effects across studies in each category. Heterogeneity was investigated visually, using the $I^{2}$ test and the Cochran test.

Results Only five trials were considered appropriate for inclusion in the meta-analysis, and these could be grouped into four categories according to mode of action. None of the studies was of more than 60 days' duration. Based on quality assessment and the short experimental period of these trials, there is currently not sufficient evidence to suggest particular efficacy of powered toothbrushes in reducing gingivitis in people who are undergoing fixed orthodontic appliance therapy.

Conclusions No conclusions can be drawn on the comparative effectiveness of powered toothbrushes in reducing gingivitis in clinical orthodontic practice. Greater standardisation of the methods used is desirable in future trials.

\section{Commentary}

Good oral hygiene during orthodontic treatment is essential to maintain the health of the dentition and supporting tissues. A common question that is asked by both orthodontists and their patients is over the efficacy of powered toothbrushes: a meta-analysis on the effectiveness of powered toothbrushes for orthodontic patients versus manual toothbrushes is therefore relevant to both the profession and the general public.

This meta-analysis has a clear and valid objective: are powered toothbrushes, for orthodontic patients, more effective than manual brushes in reducing gingival inflammation? The authors have developed a focused protocol and have followed the QUOROM guidelines ${ }^{1}$ for reporting results. The evidence-based process is precise, robust and probing. This is illustrated by the search strategy, inclusion criteria and exclusion criteria, which are well-defined, practical and allow clinical application of the results.

Within these parameters, only five trials were considered suitable for inclusion and appraisal. This is disappointing, since the maintenance of oral health during orthodontic treatment is such an important and significant area of research and clinical practice. In the excluded trials, lack of randomisation, inappropriate washout periods, unsuitable outcome measures and impractical study designs (such as the use of split-mouth trials) made them ineligible for inclusion. Unfortunately, the trials that were included in the meta-analysis were not without flaws and lacked high quality data.

Because of the weaknesses of the available data, the authors conclude that the effectiveness of powered toothbrushes at reducing gingivitis is not clear. They also draw attention to the need for greater standardisation in the methodology of future clinical trials. Currently, this is the only valid and correct conclusion to draw, even with the rigid and comprehensive method that the authors have used. Until better studies emerge, no further recommendations can be made.

\section{Practice point}

At present, the evidence is not strong enough to advocate the use of powered toothbrushes over manual toothbrushes for reducing gingivitis in orthodontic patients.

\section{Matthew Chia}

The Eastman Dental Hospital and Mayday University Hospital, London, UK.

1. Moher D, Cook DJ, Eastwood S, Olkin I, Rennie D, Stroup DF. Improving the quality of reports of meta-analyses of randomised controlled trials: the QUOROM statement. Quality of Reporting of Meta-analyses. Lancet 1999; 354:1896-1900. 